\title{
Activités de pâturage, paysages et biodiversité
}

\author{
Gérard Balent $^{\mathrm{a} *}$, Didier Alard ${ }^{\mathrm{b}}$, Vincent Blanfort ${ }^{\mathrm{c}}$, Annick Gibon ${ }^{\mathrm{a}}$ \\ a Inra-SAD, Toulouse, BP 27, 31326 Castanet-Tolosan cedex, France \\ ${ }^{\mathrm{h}}$ Université de Rouen, Ecodiv-Upresea 1293, 76821 Mont Saint-Aignan cedex, France \\ c Cirad-EMVT, 7, chemin de l'Irat, Ligne Paradis, 97410 Saint-Pierre, La Réunion, France
}

(Reçu le 12 mai 1998 ; accepté le 23 septembre 1998)

\begin{abstract}
Grazing systems, landscape patterns and biodiversity. In grazed areas, grazing activities are often the main factor that controls and influences plant and animal community dynamics. One of the new functions the society asks to the domestic herbivores is to maintain or improve the biodiversity of both plant and animal communities and the integrity of pastoral landscape as well. However, a shortage of basic knowledge characterizes the relationships between grazing activities and vegetation especially at landscape level. Grazing of vegetation by domestic herbivores is a complex hierarchical ecological process. It involves different levels of organization either in the case of free ranging animals or when feeding and spatial behaviour of grazing animals is under human control. In this paper we propose theoretical and methodological elements to help answering two important questions to improve the understanding of the relationships between grazing activity, landscape patterns and biodiversity. 1) How changes in land use management with grazing animals influence landscape patterns? 2) To which extent changes in grazing practices affect ecological characteristics of vegetation (disturbance, stability, and reversibility) ? Our skills are in the field of system analysis applied to ecology with special reference to hierarchy theory, organization of biological system theory and landscape ecology. (O) Elsevier / Inra
\end{abstract}

\section{grazing / ecological process / level of organisation / landscape patterns / biodiversity}

Résumé - Dans les espaces pâturés, le pâturage joue un rôle déterminant dans la dynamique de la diversité des espèces végétales et animales. Aujourd'hui le pâturage des animaux domestiques doit assurer le maintien de la biodiversité et de l'intégrité des paysages pastoraux. Toutefois, les effets du pâturage sur des végétations hétérogènes sont loin d'être connus. Comme tout processus écologique, le pâturage est un processus complexe à structure hiérarchique. Il importe d'identifier ses différents niveaux d'organisation spatiotemporels et ce, que les animaux choisissent librement les zones à pâturer ou qu'ils soient contraints dans leur choix par le gardiennage. Nous proposons ici quelques éléments théoriques et méthodologiques pour répondre à deux questions importantes pour décrypter les relations entre le pâturage, le territoire et la biodiversité. 1) Comment l'évolution de la gestion des espaces pastoraux ou herbagers influence-t-elle l'évolution des paysages ?2) Comment les changements de pratiques de pâturage modifient-ils les caractéristiques écologiques des formations végétales

* Correspondance et tirés à part

Tél. : 33 (0)5612852 58 ; fax : 33 (0)5 61732077 ; e-mail : balent@ telesad.toulouse.inra.fr 
pâturées ? Nos apports se situent dans le domaine de l'approche systémique appliquée aux systèmes écologiques : théorie de la hiérarchie, de l'organisation des systèmes biologiques et écologie du paysage. (C) Elsevier / Inra

\section{pâturage / processus écologique / niveau d'organisation / paysage / biodiversité}

\section{INTRODUCTION}

Les nouveaux enjeux en matière de gestion d'espèces et d'espaces par le pâturage font qu'il n'est plus possible de considérer cette activité sous le seul angle de disciplines techniques comme l'agronomie et la zootechnie. Le pâturage pour être compris et utilisé à des fins de gestion écologique doit être considéré dans des échelles spatiales et temporelles inhabituelles pour ces disciplines [24]. Aussi proposons-nous quelques éléments théoriques et méthodologiques de base couramment utilisés en écologie dès lors qu'il s'agit d'étudier le fonctionnement d'un processus écologique en insistant tout particulièrement sur le cas de la biodiversité. Nous les présentons en référence à deux questions importantes.

1) Comment l'évolution des pratiques de gestion des espaces pastoraux ou herbagers influence-t-elle l'organisation des paysages et quelles en sont les conséquences sur la biodiversité ?

2) Comment les changements de pratiques de pâturage modifient-ils les caractéristiques écologiques des formations végétales pâturées ?

Nous illustrons l'intérêt de ces concepts et méthodes sur trois terrains où les enjeux vis-à-vis du pâturage sont très contrastés : une zone de déprise agricole où le pâturage de troupeaux extensifs constitue un moyen de limiter l'enfrichement (Pyrénées centrales : [6]) ; une zone de conquête de l'espace par le pâturage au détriment de ressources végétales et animales à haute valeur patrimoniale (les Hauts de la Réunion : [10]) ; enfin une zone où le pâturage est utilisé pour partie comme un outil de gestion écologique (les pelouses calcaires des coteaux de la basse vallée de la Seine : [1]).

\section{APPORTS THÉORIQUES ET MÉTHODOLOGIQUES DE L'ÉCOLOGIE}

\subsection{La nécessité d'un modèle de référence pour le diagnostic écologique}

Pour évaluer le rôle environnemental du pâturage dans un milieu, il est nécessaire de le considérer comme un processus écologique dont on peut évaluer les effets sur un ou plusieurs systèmes écologiques (la végétation, les insectes, les oiseaux...). Cela pose la question des références à utiliser. Comment comparer deux états d'un même système écologique? Si l'on s'intéresse au cas de deux parcelles pâturées ayant des compositions botaniques statistiquement différentes (sur la base d'un test non paramétrique par exemple), sommes-nous sûrs pour autant que ces deux parcelles correspondent bien à deux réponses écologiquement différentes de la végétation au facteur pâturage ? Il se peut très bien que les différences mesurées soient dues à des espèces dont la présence ou l'absence respective dans les deux parcelles ne soit pas liée au pâturage.

Aussi, suivant en cela des réflexions déjà anciennes dans le domaine de l'écologie $[16,27]$ et plus récentes en agronomie [14], nous proposons de construire, quand cela est possible, un modèle rendant compte de la diversité des réponses d'un système écologique à la gamme de variation la plus complète possible du facteur dont on veut mesurer les effets. Il s'agit de créer une métrique écologique qui permette de mesurer une distance écologique entre deux états quelconques du système étudié plutôt qu'une distance statistique [7]. Cela sous-entend 
que, dans la modélisation d'un processus écologique complexe comme le pâturage, priorité doit être donnée à l'analyse comparative de situations contrastées sur l'expérimentation, qui retrouve ainsi son rôle essentiel d'outil de validation d'hypothèses issues d'un modèle.

\subsection{L'organisation des systèmes écologiques}

Allen et Starr [5] ont proposé d'aborder l'étude des systèmes écologiques par l'identification d'un processus écologique essentiel au fonctionnement du système étudié. Tout processus a des rythmes de comportement différents (rates of behaviour). Les changements de rythme permettent de définir les niveaux d'organisation pertinents pour rendre compte du fonctionnement du processus. Enfin, au sein de chaque niveau d'organisation, il reste à identifier les entités (ou variables) relevant du niveau considéré. Senft et al. [24] ont appliqué cette théorie à l'étude du comportement alimentaire et spatial d'herbivores sauvages. Les niveaux d'organisation sont définis sur la base des ruptures dans le rythme de décision des animaux quant au choix des activités de pâturage, depuis le rythme d'ingestion par minute au niveau de la touffe d'herbe jusqu'à la décision annuelle de migrer vers le nord ou vers le sud au rythme des saisons. À chacun des niveaux d'organisation sont associées des échelles spatio-temporelles spécifiques. Kolasa et Pickett [20] ont prolongé les bases théoriques d'Allen et Starr en proposant une théorie de l'organisation des systèmes biologiques. Elle postule qu'un système organisé est un système durable, la durabilité étant définie comme la perpétuation dynamique de la structure d'un système. Sur le plan théorique, cette proposition pose comme postulat qu'il existe un lien direct entre organisation et durabilité, question au cour des problèmes de gestion de l'espace par des herbivores. Sur le plan méthodologique, elle offre la possibilité de mesurer concrètement l'organisation qui est considérée comme la résultante de quatre propriétés composites : la hiérarchie et l'intégration, la complémentarité et la coordination. Au sein de chaque niveau d'organisation, la complémentarité est définie comme la capacité des éléments à agir simultanément pour assurer une fonction du système et la coordination comme l'ensemble des interactions entre les éléments qui leur permet de rester complémentaires.

Ainsi, un système écologique organisé ou durable, est hiérarchisé en niveaux d'organisation qui fonctionnent de manière intégrée, ce qui lui confère la capacité à résister aux changements brutaux (appelés perturbations en écologie systémique).

\subsection{D'un pool potentiel d'espèces à la biodiversité exprimée}

Le concept de biodiversité combine à la fois des échelles biologiques (du gène à l'écosystème), spatiales (du plus local au plus global) et des dimensions écologiques, fonctionnelles et structurelles [21]. Pour des raisons pratiques, les recherches sur la biodiversité portent pour la plupart sur la diversité spécifique à l'échelle des communautés [18], qui représente une dimension biologique et un compartiment fonctionnel des systèmes écologiques à la fois pertinents et faciles à appréhender.

Deux hypothèses principales explicitent les mécanismes qui permettent de passer d'un pool d'espèces potentiel à une diversité réellement exprimée :

Hypothèse 1 : la végétation exprimée en un lieu donné peut être considérée comme un sous échantillon du pool régional d'espèces qui constitue le cortège théorique potentiel de la communauté ou de l'habitat considéré (selon son échelle de définition). La biodiversité exprimée dans une communauté végétale constitue donc une composante de la biodiversité totale potentielle qui comprend à la fois la diversité non exprimée 
interne à la communauté (banque de graines) et la diversité externe représentée par le pool d'espèces présent au sein du paysage. Cela conduit à définir des unités fonctionnelles pertinentes qui permettent de rendre compte des mécanismes de remplacement des espèces en un lieu donné et qui constituent une échelle spatiale pertinente et opérationnelle pour la gestion et la restauration de la biodiversité dans ce même lieu.

Hypothèse 2 : d'une manière générale, deux grands types de gradients écologiques affectent la diversité spécifique dans les communautés végétales : ce sont les gradients liés d'une part à la disponibilité des ressources et d'autre part à la fréquence et l'intensité des perturbations [18]. L'approche prédictive repose sur l'hypothèse qu'il existe des règles d'assemblage des espèces relatives aux diverses combinaisons de ces facteurs et que la biodiversité possède par conséquent une valeur indicatrice par rapport aux facteurs environnementaux qui la contrôlent. Le rôle de ces facteurs est perçu ici comme celui d'un « filtre environnemental » qui élimine un certain nombre d'espèces à partir d'un pool théorique pour aboutir à une communauté donnée. Les règles d'assemblage prédisent quelles sont les espèces du pool théorique qui seront, du fait de leurs attributs morphologiques, écologiques ou fonctionnels, capables de passer le filtre [19].

\section{PÂTURAGE, ORGANISATION DES PAYSAGES ET BIODIVERSITÉ}

\subsection{Un modèle de référence pour rendre compte de l'évolution de l'organisation des paysages pâturés}

Les apports conjugués de la théorie de la hiérarchie et de celle de l'organisation des systèmes écologiques fournissent un cadre théorique et méthodologique pour aborder le problème de la transformation des paysages herbagers et pastoraux sous l'effet du changement des pratiques de gestion des res- sources pâturées [12]. La première étape consiste à définir un état de référence correspondant à un paysage organisé. Dans un deuxième temps on peut comparer différents paysages entre eux sur la base des différences que chacun présente avec l'état de référense [14].

Dans les Pyrénées centrales, comme dans beaucoup de zones pastorales, les paysages ont une forte valeur esthétique et patrimoniale menacée par la concentration de l'élevage sur les zones les plus favorables, l'abandon des zones les moins favorables conduisant à leur enfrichement. La question de la durabilité des relations entre élevage et paysage y est posée en terme de maintien dynamique de la structure et de la biodiversité des paysages en relation avec l'évolution des pratiques pastorales.

L'étude des pratiques anciennes de pâturage nous a conduit à proposer un modèle de gestion de l'espace par le pâturage comportant trois niveaux d'organisation [8]. Au niveau de la parcelle, le berger gère le comportement alimentaire des animaux au jour le jour en favorisant la préservation des ressources végétales ou/et l'ingestion des animaux ; l'éleveur dans son exploitation agricole gère l'ajustement entre l'offre et la demande alimentaire sur la campagne ; la société pastorale (la communauté villageoise dans le cas pyrénéen), par un corpus de règles décidées collectivement et appliquées à chacun, assure la préservation à long terme des ressources pastorales et par là même, permet au système pastoral de se reproduire, tant au plan social qu'au plan écologique.

\subsection{D'un paysage organisé}

Dans sa forme organisée (au sens de Kolasa et Pickett [20]), ce système de gestion de l'espace est fortement hiérarchique, la collectivité exerce des contraintes sur les agriculteurs et les bergers, les agriculteurs exercent des contraintes sur les bergers. Ceci permet l'intégration d'objectifs de gestion de l'espace souvent contradictoires entre 
niveaux d'organisation. La complémentarité des ressources, au sein de chaque exploitation agricole, nécessaires à leur fonctionnement n'est permise que par la coordination entre exploitations dans la gestion de ces ressources imposée par la collectivité. À cette organisation de la gestion des ressources correspond une organisation du territoire pastoral sous forme de terroirs définis comme portions d'espace de plusieurs dizaines d'hectares homogènes par leurs potentialités pédoclimatiques et leur mode d'utilisation agricole.

\section{3. À un paysage peu organisé}

Dans sa forme actuelle, ce système s'est fortement transformé suite aux différentes perturbations subies au cours du siècle écoulé (passage à l'économie de marché, dépopulation, mécanisation, quotas laitiers, nouvelle PAC). Cela a profondément modifié la structure hiérarchique du système de prise de décision concernant la gestion de l'espace pastoral. L'exploitation agricole (niveau hiérarchique intermédiaire dans le système de référence) devient le niveau principal de prise de décision, et corrélativement, le pouvoir de contrôle du niveau collectif diminue fortement ou disparaît. L'émergence des stratégies individuelles de gestion de l'espace des différentes exploitations font que complémentarité et coordination dans la gestion des ressources ont fait place à des stratégies de compétition pour les ressources clés (prés de fauche), à l'appropriation individuelle de certains terroirs de versant ou autour des quartiers de grange, et à l'abandon de parties entières du territoire progressivement envahies par des accrûs forestiers spontanés [9].

\subsection{Ou le changement de grain du paysage}

Les conséquences d'une telle évolution sur l'organisation du territoire pastoral et la dynamique des ressources végétales et des paysages concernent le changement de grain du paysage [28]. Dans un système considéré comme organisé, l'ensemble des parcelles situées dans un même terroir fait l'objet d'une gestion identique (figure l). L'hétérogénéité intra-terroir est donc très faible. En revanche, les différences entre terroirs sont importantes. Dans un système moins organisé, la tendance actuelle va vers une hétérogénéité croissante de la végétation entre les différentes parcelles d'un même terroir (gérées avec des buts différents par des agriculteurs différents) et par conséquent vers une diminution des différences dans l'état de la végétation d'un terroir à l'autre. La diversité entre les terroirs diminue, la diversité au sein des terroirs augmente.

Au cours de ce processus le paysage passe progressivement d'un gros grain à un grain fin. Au niveau d'un terroir, cela veut dire que la biodiversité augmente. À une végétation relativement homogène en raison de pratiques de gestion homogènes, succède une mosaïque de faciès végétaux répondant à la diversification des pratiques de gestion. Le cortège d'espèces animales et végétales associées à ces faciès se diversifie. Les travaux issus de l'écologie du paysage montrent toutefois que dans ce type de situation, l'augmentation du nombre d'espèces se fait le plus souvent en faveur d'espèces banales qui peuvent se contenter d'un habitat de petite taille [13]. Si on se place au niveau du paysage, le changement de grain observé aboutit à une banalisation du paysage et à une perte de biodiversité à l'exemple de la convergence de la végétation de différents terroirs d'une vallée pyrénéenne avec intensification des vallées et homogénéisation du reste du territoire [12]. Ce type d'évolution, décrit pour les Pyrénées, est très généralement répandu dans les zones de déprise agricole. Il est considéré comme un problème environnemental majeur commun aux zones de montagne européennes.

Dans de nombreuses régions, l'évolution passée de l'agriculture fait peser aujourd'hui 


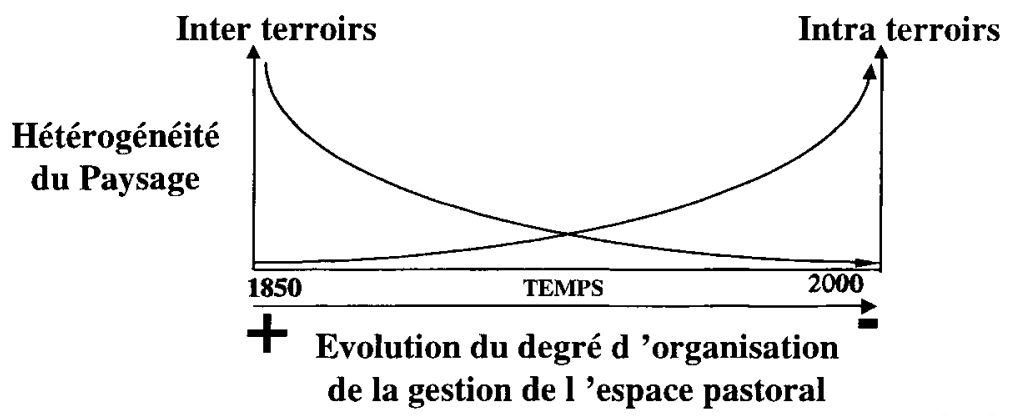

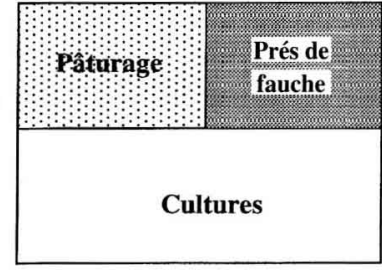

Dans le passé le territoire été structuré en terroirs organisés et homogènes dans leur utilisation PAYSAGE A GROS GRAIN

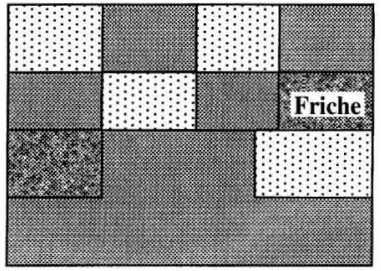

Aujourd'hui une mosaïque de parcelles diversifiées

PAYSAGE A GRAIN FIN

Figure 1. Relations entre l'évolution de l'organisation d'un territoire agricole et des caractéristiques des paysages résultants : un modèle conceptuel (d'après Balent et Gibon [9]).

des risques sur la qualité écologique et esthétique de paysages à haute valeur patrimoniale et récréative, d'où la nécessité de bien raisonner l'organisation du pâturage à des niveaux d'organisation élevés (un territoire, l'ensemble des agriculteurs qui l'utilisent).

Dans la vallée de la Seine, la même dynamique de changement de l'organisation des paysages s'est produite. La diversité des modes d'occupation du sol devient plus grande au sein des anciens terroirs qu'entre ces mêmes terroirs [23]. Ces terroirs abritent des espèces à haute valeur patrimoniale (plusieurs espèces d'orchidées) ayant une faible amplitude d'habitat, c'est-à-dire correspondant à un type particulier de combinaison entre pratiques de pâturage et milieu. Ils constituent les unités fonctionnelles de ces populations spécialisées, où s'effectuent par conséquent l'ensemble des étapes du cycle biologique de ces espèces [2]. L'augmentation de la diversité intra-terroirs cor- respond pour ces espèces, inféodées au substrat calcaire, à une fragmentation de leur habitat potentiel et traduit l'apparition de pratiques incompatibles avec leur survie (intensification ou déprise). Le terroir devient, pour l'espèce, une mosaïque changeante où les « taches d'habitats propices » varient dans l'espace et le temps. Le rôle jusque là prépondérant des pratiques agricoles dans le filtrage du pool d'espèces disponible s'estompe peu à peu au profit des facteurs paysagers (ceux qui contrôlent la dispersion) et des stratégies de persistance des espèces dans le milieu (graine ou bulbe). Cela menace la survie dans le paysage à la fois des espèces filtrées par les pratiques agricoles et par les facteurs paysagers.

Cet exemple permet de poser la question du bien fondé de la multiplication d'actions de conservation d'espèces ou de gestion de milieu par le pâturage conduites à la parcelle ou sur un groupe de parcelles, alors 
que le fonctionnement des processus que l'on cherche à contrôler s'inscrit dans des unités de paysage plus vastes.

\section{PÂTURAGE, ORGANISATION DE LA VÉGÉTATION ET BIODIVERSITÉ}

\subsection{Diversité et stabilité de la végétation pâturée : exemple des Pyrénées}

On considère en général que le pâturage augmente la diversité spécifique des prairies quand son intensité est moyenne et qu'il diminue cette diversité spécifique quand son intensité augmente. Si l'on suit les conclusions de Tilman [25] sur la relation directe entre la diversité spécifique et la capacité d'une végétation à résister à des perturbations (invasion d'espèces exogènes), cela voudrait dire qu'aux intensités moyennes de pâturage la végétation est plus stable qu'aux intensités élevées. Cela n'est pas sans implications pratiques.

Dans les Pyrénées nous avons modélisé les réponses de la végétation des prairies aux variations d'intensité de deux gradients écologiques, la fertilité et l'intensité de pâtu- rage. La modélisation est basée sur une représentation simultanée de la variance inter relevée et intra relevée de la composition botanique le long d'un gradient écologique. Ces deux variances représentent respectivement l'ordination et l'organisation des communautés végétales le long de ce gradient [6]. La diversité intra relevé est relative à la distribution des espèces qui composent ce relevé le long du gradient. Elle est faible quand les espèces sont voisines sur le gradient (communauté homogène); elle est forte quand les espèces sont éloignées (communauté hétérogène). Une forte diversité intra reflète un degré élevé de perturbation de la végétation.

Les résultats présentés sur la figure 2 concernent le gradient de pâturage. Nos données confirment l'effet dépressif du pâturage sur la richesse spécifique qui passe d'une cinquantaine à une vingtaine d'espèces quand le prélèvement par les animaux passe de 0,5 à 5 tonnes de MS/ha/an. Parallèlement la diversité intra relevé est peu affectée par l'augmentation de la pression de pâturage sauf pour les valeurs les plus fortes, où la création de micro-perturbations dans le tapis végétal permet à des espèces envahissantes de s'installer. Ces résultats montrent
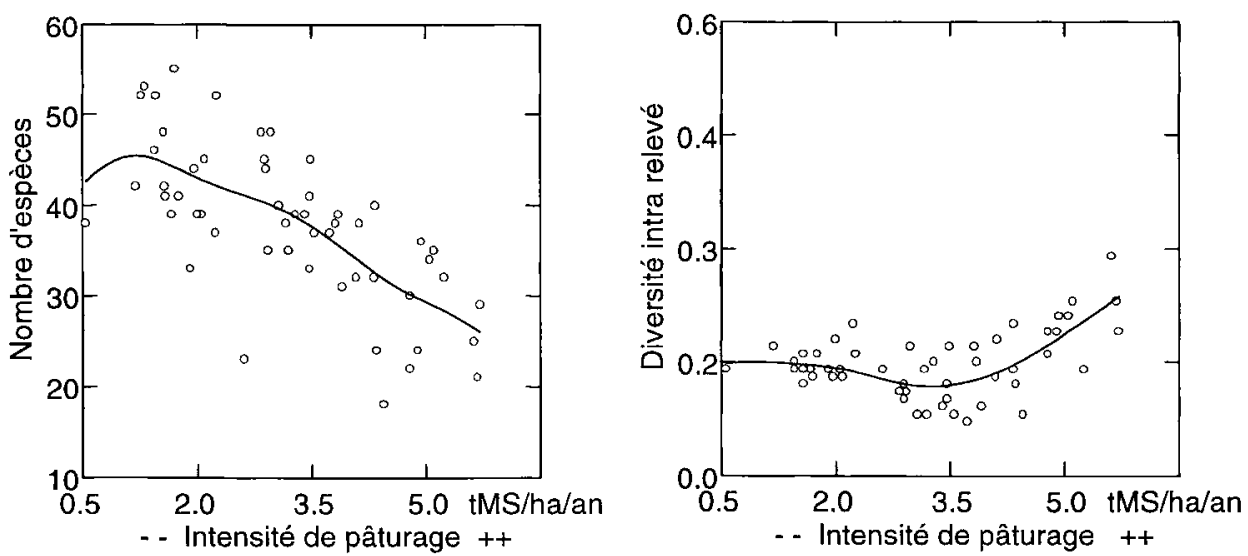

Figure 2. Relations entre intensité du pâturage, diversité spécifique et organisation de la végétation. Le lissage des courbes est réalisé par la méthode DWLS puissance 0,85. 
que suivant la façon dont on rend compte de la biodiversité les conclusions sont différentes. Avec une évaluation structurelle (richesse spécifique), le pâturage affecte fortement la biodiversité ; avec une vision fonctionnelle (diversité intra-organisation) le pâturage a peu d'effets sauf pour les très fortes intensités, pour lesquelles il perturbe l'organisation de la végétation.

\subsection{Réversibilité de la dynamique de végétation engendrée par le pâturage : exemple de l'île de la Réunion}

Un des problèmes clé dans le contrôle de la dynamique de la végétation prairiale est l'ajustement de la quantité prélevée à la quantité produite et ce, quel que soit le niveau de production de la parcelle. La sous consommation d'herbe est un facteur de dégradation de la végétation au même titre que le surpâturage. Dans des situations de déprise l'accumulation d'herbe conduit à l'envahissement des pâturages par des espèces ligneuses. Dans des milieux très productifs comme les pâturages tropicaux humides la sous consommation chronique pendant la saison des pluies conduit au même phénomène.

Dans les zones d'altitude de l'île de la Réunion où se développent actuellement des systèmes d'élevage bovin lait et allaitant basés sur l'utilisation intensive de pâturages créés de toute pièce au dépend de la végétation naturelle, ces phénomènes d'envahissement sont très importants. Dans ce contexte insulaire où la diversité biologique de la végétation et sa valeur patrimoniale sont remarquables (fort taux d'endémisme) le caractère réversible ou non de ce processus est essentiel à connaître [10].

Dans les Hauts de l'ouest par exemple (figure 3) les pâturages actuels sont établis sur d'anciennes jachères à tamarin des hauts (Acacia heterophylla), espèce forestière endémique, qui résultent elles-mêmes de la mise en culture de landes à Philippia montana. Dans les situations les moins intensifiées, les pâturages sont actuellement dominés par la houlque laineuse (Holcus lanatus). En cas de sous utilisation, ces formations à houlque peuvent être à nouveau colonisées par le tamarin des hauts. Une première voie d'intensification des prairies à houlque se fait par introduction du kikuyu (Pennisetum clandestinum) plante stolonifère tropicale très agressive qui, en général, occupe rapidement l'ensemble de la parcelle. Une autre voie consiste à implanter des prairies à dactyle et ray-grass anglais. La sous-consommation chronique observée en saison des pluies conduit à l'envahissement de ces prairies par le kikuyu aux altitudes les plus basses et par l'Acacia mearnsii (arbre exotique australien utilisé jadis dans les jachères) aux altitudes élevées. Les parcelles de kikuyu sous utilisées sont, elles aussi, envahies par Acacia mearnsii qui semble ainsi constituer un stade ultime de dégradation des prairies intensives dont on ne connait aujourd'hui aucun exemple de réversibilité. Seules les parcelles de dactyle et ray-grass situées dans des paysages où kikuyu et acacia sont absents peuvent évoluer vers des formations sub-spontanées à houlque. Dans ce milieu tropical insulaire où le taux d'endémisme est élevé, des pratiques de pâturage mal maîtrisées favorisent le développement d'espèces envahissantes le plus souvent exotiques qui empêchent dans la majorité des cas le retour vers la végétation naturelle, et constituent un danger pour la conservation du patrimoine génétique local.

\section{CONCLUSIONS}

Vouloir utiliser le pâturage comme outil de gestion et de contrôle de formations végétales complexes oblige à situer son étude dans le champ de l'écologie systémique [26]. Ceci implique la construction d'un modèle conceptuel permettant de rendre compte de la diversité des relations pâturage végéta- 


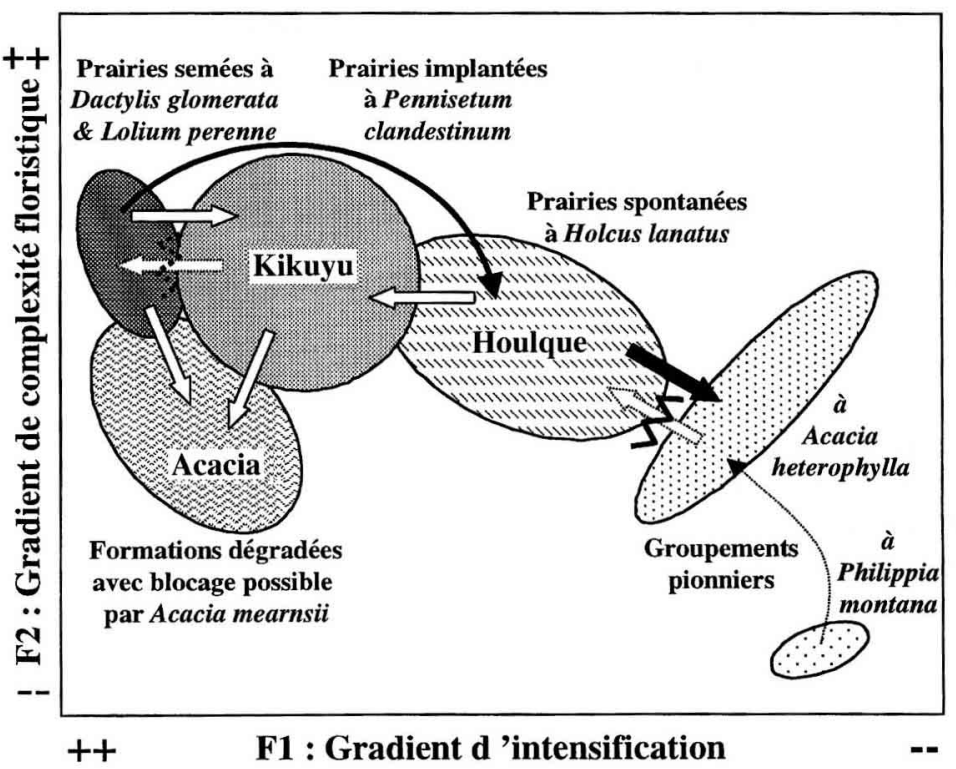

Type de trajectoires

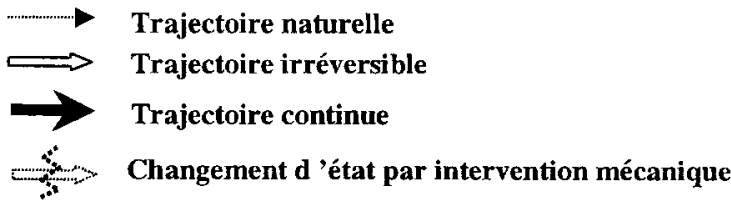

Figure 3. Trajectoires d'évolution de la végétation des formations pâturées dans l'île de la Réunion : l'exemple des Hauts de l'ouest. Le plan factoriel regroupe chacun plus de 100 relevés botaniques traités par analyse des correspondances. Seules sont représentées les ellipses (intervalle de confiance 0,75 à 0,9 ) entourant les types de végétation issus d'une classification ascendante hiérarchique sur les premiers facteurs de l'analyse des correspondances. Les trajectoires sont ajustées à l'ocil.

tion, l'identification des processus écologiques majeurs qui structurent les relations pâturage végétation, et pour chaque processus l'identification des niveaux d'organisation pertinents pour rendre compte de leur fonctionnement. Ceci sous-entend de développer des travaux sur une large gamme d'échelles d'espace et de temps [11].

Si l'approche systémique des processus écologiques est une nécessité, elle ne va pas sans difficulté. Une des difficultés majeures concerne le problème de l'adéquation entre les niveaux d'observation et les niveaux de fonctionnement d'un processus écologique
$[3,4]$. Nous l'avons dit, la majorité des observations sur les relations entre pâturage, végétation et biodiversité concernent des communautés végétales et des pratiques enregistrées au niveau de la parcelle. La parcelle est un niveau d'observation commode et incontournable mais ni la communauté, ni la parcelle ne sont des niveaux pertinents et suffisants pour comprendre les relations paysage, pâturage et biodiversité. Un des enjeux majeurs aujourd'hui, au coeur des problématiques de l'écologie du paysage, est d'établir des correspondances entre les niveaux d'organisation de fonctionnement des pro- 
cessus écologiques, en général mal connus, et les niveaux d'organisation de la gestion de ces mêmes processus par les activités agricoles, en général bien connus.

Les bases théoriques et les exemples apportés suggèrent qu'un effort de révision des problématiques doit être entrepris dans la façon d'aborder les processus biologiques concernés [24]. Coté herbivore, parallèlement aux recherches sur l'ingestion qui concernent les effets de la végétation sur l'animal, et qui, par conséquent, ne permettent pas de répondre à la question de la gestion de territoires hétérogènes par des herbivores, l'effort doit porter sur l'étude in situ des déterminants du comportement alimentaire et spatial et de ses effets sur la végétation, domaine dans lequel les résultats de la littérature sont encore très contradictoires [22]. Coté végétation, les besoins de références concernent essentiellement l'étude des effets du pâturage sur les stratégies de reproduction et de dissémination des espèces au niveau des populations, et sur les phénomènes de compétition interspécifique pour les différentes ressources au niveau des communautés prairiales.

Une telle évolution des recherches suppose de prendre en compte les modalités d'organisation spatiale de la gestion des espaces pâturés au niveau des paysages et des territoires [12] ainsi que l'articulation de ces niveaux avec ceux plus familiers aux agronomes et aux zootechniciens [15]. Aussi, vouloir gérer la biodiversité par le pâturage demande d'intégrer dans la réflexion non seulement les connaissances sur les aspects biotechniques de l'utilisation du pâturage, mais aussi celles relatives à son organisation sociale, qu'il s'agisse de la gestion d'une exploitation d'élevage ou de l'organisation entre acteurs au niveau de la gestion d'un territoire [17]. Ainsi la gestion de la biodiversité n'est elle pas dissociable de la problématique plus générale d'une agriculture durable dans ses dimensions socio-économiques.

\section{RÉFÉRENCES}

[1] Alard D., La végétation pastorale de Normandie centrale. Phyto-écologie, agronomie et dynamique. Conséquences pour la gestion d'un espace agricole en mutation, thèse, Université de Rouen, 1990.

[2] Alard D., Poudevigne I., Les facteurs de contrôle de la biodiversité dans un paysage rural : une approche agro-écologique, Ecologie 28 (1997) 25-38.

[3] Allen T.F.H., Hoekstra T.W., The confusion between scale-defined levels and conventional levels of organisation in ecology, J. Veg. Sci. 1 (1990) 5-12.

[4] Allen T.F.H., O’Neill, R.V., Hoekstra, T.W., Interlevel relations in ecological research and management: some working principles from hierarchy theory, J. Appl. Syst. Anal. 14 (1987) 63-79.

[5] Allen T.F.H., Starr T.B., Hierarchy. Perspectives for ecological complexity, University of Chicago Press, Chicago, II, 1982.

[6] Balent G., Construction of a reference frame for studying the changes in species composition in grassland, Options Méditerranéennes 15 (1991) 73-81.

17] Balent G., La qualité des systèmes écologiques : Le point de vue de l'écologue, Études et Recherches sur les Systèmes Agraires et le Développement 28 (1994) 259-266.

[8] Balent G., Gibon, A., Définition et représentation du système pastoral. Niveaux d'organisation et pratiques de pâturage, Études et Recherches sur les Systèmes Agraires et le Développement 11 (1988) 65-78.

[9] Balent G., Gibon A., Organisation collective et individuelle dans la gestion des ressources pastorales : conséquences sur la durabilité agroécologique des ressources, in: N.P. Zervas, Hatziminaoglou J. (éds.), The optimal exploitation of marginal Mediterranean areas by extensive ruminant production systems, EAAP Publication $n^{\circ} 83$, Thessalonique, 1996, pp. 365-375.

[10] Blanfort V., Agro-écologie des pâturages d'altitude à l'île de la Réunion. Pratiques d'éleveurs et durabilité des ressources herbagères dans un milieu à fortes contraintes, thèse, Université d'Orsay, 1996.

111] Brown B.J., Allen T.F.H., The importance of scale in evaluating herbivory impact, Oikos 54 (1989) 189-194.

112| Di Pictro F.. Balent G., Dynamique des pratiques pastorales et des paysages : une approche pluri-échelles appliquée aux Pyrénées ariégeoises (France), Agronomic 17 (1997) 1.39-155.

113] Forman R.T.T., Land Mosaics. The ecology of landscapes and regions, Cambridge University Press, Cambridge, 1995. 
[14] Fresco L.O., Kroonenberg S.B., Time and spatial scales in ecological sustainability, Land Use Policy (July 1992) 155-168.

[15] Gibon A., Addressing Livestock Farming Systems ecological sustainability at the regional level: a example from the Central Pyrenees, in: Sorensen J.T. (Ed.), Livestock Farming Systems: More than food production, Wageningen Pers, EAAP Publ. 89 (1997) 30-41.

[16] Holling C.S., Resilience and stability of ecological systems, Annu. Rev. Ecol. Syst. 4 (1973) j-23.

[17] Hubert, B., Girault N., De la touffe d'herbe au paysage, Inra Publications, Versailles, 1988.

[18] Huston M.A., Biological Diversity, The coexistence of species on changing landscapes, Cambridge University Press, Cambridge, 1994.

[19] Keddy P.A., Assembly and response rules: two goals for predictive community ecology, J. Veg. Sci. 3 (1992) 157-164.

[20] Kolasa J., Pickett S.T.A., Ecological systems and the concept of biological organization, Proc. Natl. Acad. Sci. USA, 86 (1989) 8837-8841.

[21] Noss R.F., Indicators for monitoring biodiversity: A hierarchical approach, Conserv. Biol. 4 (1990) 355-364.
[22] Olff H., Ritchie M.E., Effects of herbivores on grassland plant diversity, Trends Ecol, Evol. 13 (1998) 261-265.

[23] Poudevigne I., Van Rooij S.A.M., Morley C.R., Alard D., Dynamics of rural landscapes and their main driving factors: a case study in the Seine valley, Normandy, France, Lands. Urb. Plann. 38 (1997) 93-103.

[24] Senft R.L., Coughenour M.B., Bailey D.W., Rittenhouse L.R., Sala O.E., Swift D.M., Large herbivore foraging and ecological hierarchies. Landscape ecology can enhance traditional foraging theory. BioScience 37 (1987) 789-799.

[25] Tilman D., Community invasibility, recruitment limitation, and grassland biodiversity, Ecology 78 (1997) 8I-92.

[26] Van Dyne G. M., A systems approach to grasslands, in: Norman M.J.T. (éd.), Proc. XI International Grassland Congress, Surfers Paradise, Queensland, Australia, 1970, pp. A131-A143.

[27] Whittaker R.H., Gradient analysis of vegetation, Biol. Rev. 42 (1967) 207-264.

[28] Wiens J.A., Spatial scaling in ecology, Funct. Ecol. 3 (1989) 385-399. 